\title{
In-situ measurement of the $\beta$ to $\alpha$ phase transformation kinetics in a Ti-5553 alloy using laser ultrasonics
}

\author{
M. C. M. Rodrigues ${ }^{\mathrm{a}, *}$, T. Garcin ${ }^{\mathrm{b}}$, M. Militzer ${ }^{\mathrm{a}}$ \\ a The Centre for Metallurgical Process Engineering, The University of British Columbia, 309-6350 Stores Rd., Vancouver, BC, \\ Canada V6T 1 Z4 \\ b Tecnar Automation Ltée, 1321 Rue Hocquart, Saint-Bruno-de-Montarville, QC, Canada J3V 6B5 \\ *mariana.rodrigues@alumni.ubc.ca
}

\begin{abstract}
$\underline{\text { Abstract }}$
Ti-5553 alloy (Ti-5Al-5Mo-5V-3Cr wt.\%) is one of the newest high strength metastable $\beta$-titanium alloys to be used in large section components of aircraft structures. Like other Ti-alloys, Ti-5553 can be strengthened by precipitation of the $\alpha$-phase during aging heat treatments. In the present study, we explored the potential of laser ultrasonics for metallurgy (LUMet) as an in-situ tool to measure the $\alpha$ precipitation kinetics during high temperature isothermal aging in a Ti-5553 alloy. The observed change in longitudinal ultrasound velocity was seen to be linearly correlated with the $\alpha$-phase fraction measured via post-mortem electron microscopy. The results are promising for a wider use of LUMet to design optimized heat treatment schedules for commercial Tialloys. Laser ultrasonics offers the potential for real-time monitoring of microstructure evolution in industrial production lines.
\end{abstract}

\section{Introduction}

Metastable $\beta$-titanium alloys have been increasingly used in the aerospace industry due to their wide range of tensile and fatigue strength combinations, in addition to their light weight and high corrosion resistance [1, 2]. In particular, Ti-5553 (Ti-5Al$5 \mathrm{Mo}-5 \mathrm{~V}-3 \mathrm{Cr} w \mathrm{t} . \%$ ) has gained interest in aircraft applications, such as in the design of landing gears. In these parts, Ti-5553 has been gradually replacing Ti-1023 (Ti-10V-2Fe-3Al wt.\%), due to its higher tensile strength, wider processing window and deeper hardenability, making it the preferred alloy for thick section forgings [3]. The main strengthening mechanism in Ti-5553 results from the formation of fine $\alpha$-phase precipitates during aging in the $\alpha+\beta$ phase field, which is typically conducted after a $\beta$ solid solution treatment. The gain in mechanical properties is directly influenced by the size, morphology, distribution and volume fraction of the $\alpha$ precipitates $[4,5]$. Therefore, it is industrially relevant and of scientific interest to quantify the $\alpha$ precipitation kinetics during aging.

Although widely used in steels, conventional dilatometry for phase transformation measurements is not well adapted in Ti-alloys for which the measurement is affected not only by changes in crystal structure, but also by the partitioning kinetics of substitutional alloying elements [6]. Moreover, the phase transformation in Ti-alloys is associated with a relatively small volumetric change which usually falls below the detection limit of most dilatometry equipment [7, 8]. Alternative techniques include synchrotron X-ray diffraction which, however, is limited by the fast growth of $\beta$ grains that often occurs during the $\beta$ solution treatment. The bias introduced by the low grain sampling statistics results in challenging interpretation of the diffraction ray intensity [9]. Furthermore, due to the low penetration depth of X-rays, results can be affected by the formation of $\alpha$-casing at high temperatures [10]. While neutron penetration is deeper, neutron diffraction is limited in terms of acquisition time when measuring phase transitions in pure titanium [11], i.e. the fast rate of transformation does not allow for the construction of a complete scan. Finally, in-situ electrical resistivity measurements are suitable to evaluate transformation kinetics in Ti-alloys although correlations with other techniques remain necessary for a quantitative analysis with respect to absolute phase fractions [9, $12,13]$.

Laser ultrasonics sensing (LUS) has been identified as a useful tool to measure phase transformations in metals and alloys [14-19]. In addition to being in-situ, it is a non-contact and non-destructive technique that permits real-time tracking of microstructure evolution during complex thermomechanical processing, which is advantageous from the industrial perspective. Phase transitions can be determined by this technique because the ultrasound velocity depends on the elastic properties and density of the investigated material [20], which typically change with phase fractions and crystallographic texture. Although LUS has been developed in the past three decades, there is still a limited number of publications on the usage of this technique for phase transformation measurements in titanium and Ti-alloys [17-19]. Zamiri et al. [17] observed the sensitivity of LUS in detecting phase transitions in a Ti-6Al-4V wt.\% alloy during continuous heating. Attempts to validate LUS measurements in Ti-systems were later done by Hinterlechner et al. [19]. Aided by synchrotron X-ray diffraction, a good agreement was observed between the change in wave velocity and the $\alpha$ dissolution during continuous heating of an $\alpha+\beta$ Ti-alloy [19]. However, investigations on the complete $\alpha+\beta \rightarrow \beta$ transformation were not performed by Hinterlechner et al. [19]. Variation in ultrasound velocity upon allotropic transformation was observed by Shinbine et al. [18] during thermal cycling of pure titanium. Here, the velocity behavior was modeled assuming that its change is correlated to a volume-fraction-weighted average of individual constituents by a simple linear addition law, as proposed by Kruger and Damm for steels [16]. But to date no validation of this rule of mixtures was conducted for titanium and its alloys.

The present work aims to investigate the relationship between ultrasound velocity and $\alpha$ phase fraction in a Ti-5553 alloy. This alloy was selected for its current industrial relevance and its relatively long transformation times due to the presence of slow diffusing alloying elements. Hence, the $\beta$-phase can be retained at room temperature at reasonable cooling rates $\left(>0.25^{\circ} \mathrm{C} / \mathrm{s}\right)[21]$. Following a $\beta$ solution treatment, the $\alpha$-phase precipitation behavior was studied by isothermal treatment at $600{ }^{\circ} \mathrm{C}$. Data 
acquisition was carried out during the isothermal aging and the observed change in wave velocity was correlated with the $\alpha$ volume fraction, measured by ex-situ metallography.

\section{$\underline{2 \text { Material and experiments }}$}

A Ti-5553 alloy was used in this study as provided by UTC Aerospace Systems in the as-forged condition with the chemical composition shown in Table 1 . The $\beta$-transus temperature $\left(\mathrm{T}_{\beta}\right)$ of this system is $\sim 840{ }^{\circ} \mathrm{C}[22]$ and its molybdenum equivalent $\left([\mathrm{Mo}]_{\text {eq }}\right)$ is 12.7 as calculated using the empirical equation proposed by Collings [23].

Table 1: Chemical composition of the Ti-5Al-5V-5Mo-3Cr alloy (wt.\%)

\begin{tabular}{ccccccccccc}
\hline $\mathbf{A l}$ & $\mathbf{V}$ & $\mathbf{M o}$ & $\mathbf{C r}$ & $\mathbf{F e}$ & $\mathbf{S i}$ & $\mathbf{Z r}$ & $\mathbf{O}$ & $\mathbf{H}$ & $\mathbf{C}$ & $\mathbf{T i}$ \\
\hline 5.39 & 5.01 & 5.03 & 2.80 & 0.32 & 0.03 & 0.01 & 0.15 & 0.002 & 0.01 & Bal. \\
\hline
\end{tabular}

Specimens with dimensions of $60 \times 10 \times 3 \mathrm{~mm}$ were machined from the as-forged material to perform the tests. Heat treatments as well as laser ultrasonics measurements were carried out in a Gleeble 3500 thermomechanical simulator (Dynamic System Inc. Poestenkill, NY) coupled with a LUMet sensor. Unlike in conventional transducer contact methods, the ultrasound pulse is generated by ablating a fine surface layer of the sample by a frequency-doubled Q-switched Nd:YAG laser, which has a wavelength of $532 \mathrm{~nm}$, a pulse duration of $6 \mathrm{~ns}$ and a maximum energy of $72 \mathrm{~mJ}$. The created ultrasound wave propagates back and forth through the sample. Consecutive arrivals of the pulse at the sample surface create a small surface displacement that modulates the detection laser light reflected on the sample. The detection laser is a frequency-stabilized Nd:YAG pulsed laser which illuminates the generation spot with infrared radiation $(1064 \mathrm{~nm})$ and with a pulse duration of $90 \mu$ s. The reflected light is demodulated using a photo-refractive crystal and the ultrasound signal with an amplitude proportional to the ultrasound surface displacement is collected. Ultrasound velocity is given by the ratio of the propagation distance and the time between two echoes, which is calculated using a cross correlation function between two selected echoes. In this case, the ultrasound bandwidth ranges from 2 to $10 \mathrm{MHz}$ with a highest signal to noise ratio centered around $5 \mathrm{MHz}$. K-type thermocouples, spot-welded at the center of the specimen on the opposite side of the laser beam, were used to measure the specimen temperature. The tests were conducted under a vacuum atmosphere of $\sim 0.002 \mathrm{~Pa}$.

The first heat treatment consisted of a $\beta$-solution treatment at $900{ }^{\circ} \mathrm{C}$ for $15 \mathrm{~min}$ followed by controlled cooling at a rate of $6.7^{\circ} \mathrm{C} / \mathrm{s}$ to room temperature. This test was conducted to validate the complete dissolution of the $\alpha$ phase prior to the isothermal aging treatment. The LUMet signal was acquired during cooling from $900{ }^{\circ} \mathrm{C}$ to $450{ }^{\circ} \mathrm{C}$. The $\omega$-solvus temperature has been determined to be in the range of 350-400 ${ }^{\circ} \mathrm{C}$ for this alloy [24]. Therefore, samples rapidly cooled from the $\beta$ phase-field to temperatures above $400{ }^{\circ} \mathrm{C}$ would not undergo any athermal or isothermal $\omega$ precipitation. Subsequently, the $\alpha$ precipitation kinetics of the alloy was investigated during aging at $600{ }^{\circ} \mathrm{C}$, i.e. above the temperature range where the hexagonal $\omega$ phase can form. Samples for the aging treatment were cooled at $6.7{ }^{\circ} \mathrm{C} / \mathrm{s}$ from the $\beta$ solutionizing temperature $\left(900{ }^{\circ} \mathrm{C}\right)$ to the aging temperature $\left(600^{\circ} \mathrm{C}\right)$. To assess the change in wave velocity with the $\alpha$-phase fraction, samples were quenched using He gas after different aging times.

Specimens for scanning electron microscopy (SEM) and electron backscatter diffraction (EBSD) characterization were prepared by standard metallographic techniques comprising of mechanical surface grinding and polishing. The finishing step consisted of mechanical polishing with a 5:1 volume mixture of $0.05 \mu \mathrm{m}$ colloidal silica and $30 \mathrm{wt} . \%$ hydrogen peroxide solution. A Zeiss Sigma field emission scanning electron microscope at $20 \mathrm{kV}$ accelerating voltage was used for EBSD phase mapping and backscattered imaging (BSE). EBSD data acquisition was performed on selected samples using an EDAX DigiView Camera and TSL Orientation Imaging Microscopy (OIM) software at a step size of $0.05 \mu \mathrm{m}$. Phase maps were obtained by means of the TSL OIM Analysis 6.2 software.

The atomic number contrast in BSE images was used to quantify the $\alpha$ volume fractions via image analysis. Uneven image background, originating from different $\beta$ grain orientations and redistribution of alloying elements with time, was corrected in order to set a single threshold grey value for all conditions. For this purpose, the so-called rolling ball algorithm [25] using a morphological closing approach was implemented in MATLAB. Here, a criterion to find the best ball radius based on image magnification and foreground feature size was used. Before applying the algorithm, the 8-bit images were normalized to set the greyscale histogram distributions from 0 to 255 in all images. The threshold grey value was set such that the $\alpha$ fraction of a selected BSE image, taken at the exact same area at which an EBSD scanning was conducted, matched with the $\alpha$ fraction obtained from the corresponding EBSD phase map. This value was then used in all the images taken for different holding times. The $\alpha$ phase fractions were measured from at least 10 images in each condition. All SEM parameters that could affect phase contrast, such as working distance, beam current, etc., were kept constant.

\section{$\underline{3 \text { Results and discussion }}$}

An SEM micrograph of the Ti-5553 alloy in the as-received condition is shown in Fig. 1a. Its microstructure consists of a duplex structure of globular and acicular $\alpha$-phase constituents dispersed in the $\beta$ matrix, typically produced during conventional $\alpha+\beta$ forging. Fig. $1 \mathrm{~b}$ shows the change in longitudinal wave velocity $\left(\Delta \mathrm{V}_{\mathrm{L}}\right)$ during controlled cooling at $6.7^{\circ} \mathrm{C} / \mathrm{s}$ following the $\beta$ solution treatment at $900{ }^{\circ} \mathrm{C}$ for $15 \mathrm{~min}$. Each data point in the graph corresponds to one ultrasound measurement and there is a very good repeatability in the three investigated samples. The velocity increases linearly with decreasing temperature due to changes in elasticity and density of the alloy as the temperature decreases [20]. The monotonic velocity increase also indicates that no $\beta \rightarrow \alpha+\beta$ transformation occurred during cooling at $6.7^{\circ} \mathrm{C} / \mathrm{s}$, as confirmed with SEM micrographs that show a fully metastable $\beta$ microstructure with a mean equivalent area diameter (EQAD) of $182 \mu \mathrm{m}$ (Fig. 1c). From the LUMet results, the temperature dependence of ultrasonic velocity in the $\beta$-phase is found to be $-3.7 \times 10^{-4} \mathrm{~mm} . \mu \mathrm{s}^{-1} .{ }^{\circ} \mathrm{C}^{-1}$. 
a)

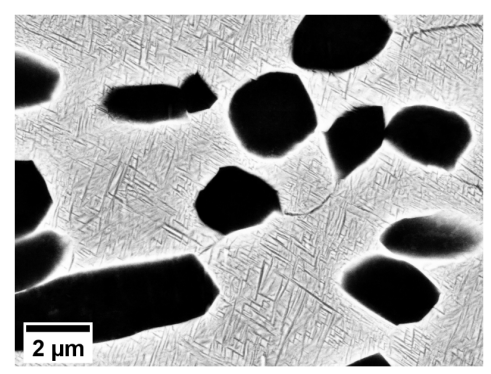

b)

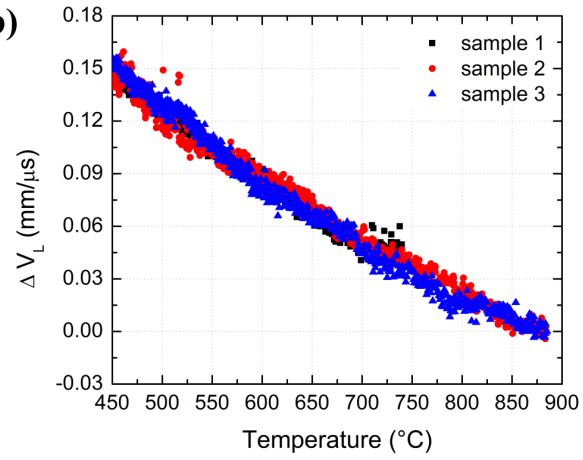

c)

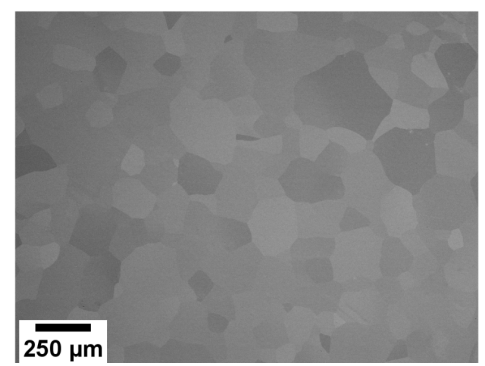

Fig. 1: Ti-5553 alloy a) SEM/BSE image of the as-received microstructure; b) Change in longitudinal wave velocity in the fully $\beta$ condition during continuous cooling; c) SEM/BSE image of the as-cooled microstructure

The above analysis also indicates that there is a statistically sufficient number of $\beta$ grains in the volume probed by the ultrasound pulse given by the laser spot diameter of $2 \mathrm{~mm}$ and the sample thickness of $3 \mathrm{~mm}$. Assuming a spherical grain approximation, the number of $\beta$ grains is estimated to be of order 3000 which is sufficient to presume a random grain orientation distribution in the probed volume [26]. This constitutes a critical aspect of the LUMet measurements to minimize potential effects of $\beta$ crystallographic orientation on the ultrasound velocities, as the elastic constants in the bcc phase are highly anisotropic [18, 22].

Fig. 2 shows the change in longitudinal wave velocity during aging at $600{ }^{\circ} \mathrm{C}$ as a function of aging time. $\mathrm{V}_{\mathrm{t}}$ is the absolute velocity at a given time " $\mathrm{t}$ " and $\mathrm{V}_{0}$ was the velocity measured at the onset of the isothermal holding, i.e. in the fully $\beta$ condition.

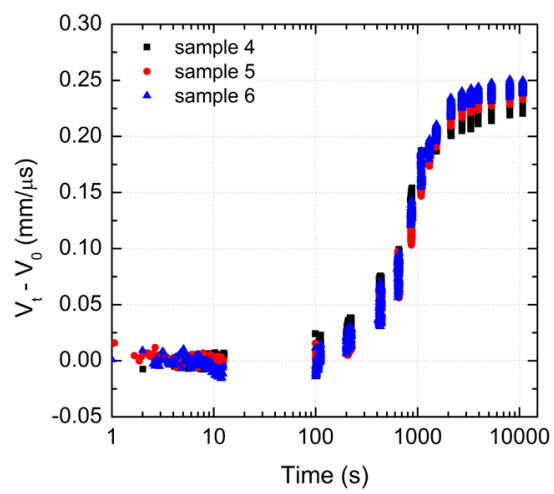

Fig. 2: Change in ultrasound velocity in Ti-5553 during $3 \mathrm{~h}$ holding at $600{ }^{\circ} \mathrm{C}$

Ultrasonic velocity can be affected, at different order of magnitudes, by porosity, phase transformations, crystallographic texture, grain size, internal friction, temperature and stress [27]. At constant temperatures without deformation and operating in the Rayleigh regime (i.e. the mean grain size is much smaller than the ultrasound wavelength $\lambda$ ), the wave velocity is primarily influenced by phase transformation and crystallographic texture. Using the classical wave equation $\lambda=v / f$, where $\mathrm{V}$ is the longitudinal ultrasonic velocity in the $\beta$-phase $(4600 \mathrm{~m} / \mathrm{s})$ [17] and $\mathrm{f}$ is the centered frequency of the broadband ultrasound (5 $\mathrm{MHz}$ ), the ultrasound wavelength is estimated to be $920 \mu \mathrm{m}$, i.e. the wavelength is about 5 times larger than the mean $\beta$ grain size $(182 \mu \mathrm{m})$ at the beginning of aging such that the measurements are indeed conducted in the Rayleigh scattering regime. As there is a statistically sufficient number of grains in the probed volume any observed velocity change can thus be attributed to phase transformation.

Indeed, Fig. 2 shows that the change in velocity measured during aging depicts a sigmoidal shape, which is consistent with a classical diffusion controlled nucleation-growth type phase transformation kinetics. A relatively good test repeatability among the three measured samples is again observed. The variation among repeated experiments is estimated around $0.02 \mathrm{~mm} / \mu \mathrm{s}$ before the transformation and can be as high as $0.05 \mathrm{~mm} / \mu \mathrm{s}$ when both phases, $\alpha$ and $\beta$, are present. In particular, during the transformation the decreased precision may be related to the redistribution of alloying elements that may affect elastic properties and resulting wave velocities. 
The results from ex-situ metallography of samples quenched from $600{ }^{\circ} \mathrm{C}$ after different holding times were used to construct a calibration curve of the measured $\alpha$-phase fraction as a function of the change in velocity. Here, EBSD was used to measure the $\alpha$ fraction for the longest holding time of 3 hours where equilibrium is approached. The $\alpha$ fraction measured by EBSD is then used to select the threshold grey value to systematically measure the amount of $\alpha$-phase from BSE images, as described in section 2. Fig. 3a shows a BSE image of the sample aged for 3 hours and rapidly cooled to room temperature, while Fig. $3 \mathrm{~b}$ shows the corresponding EBSD phase map at the same area. The microstructure seen in Fig. 3 consists of fine $\alpha$ variants homogeneously dispersed in a basketweave fashion within the $\beta$ matrix. This type of arrangement is commonly seen in $\alpha+\beta$ and metastable $\beta$ Tialloys and results from the tendency of the system to minimize the total $\beta \rightarrow \alpha$ transformation shape strain and the corresponding strain energy by clustering of multiple $\alpha$ lath variants, i.e. the principle of self-accommodation [28]. The $\alpha$-phase fraction (in \%) is measured to be $59 \% \pm 1 \%$ which is in good agreement with the $\alpha$-phase equilibrium fraction of $60 \%$ calculated using the TTTI3 database in ThermoCalc.

a)

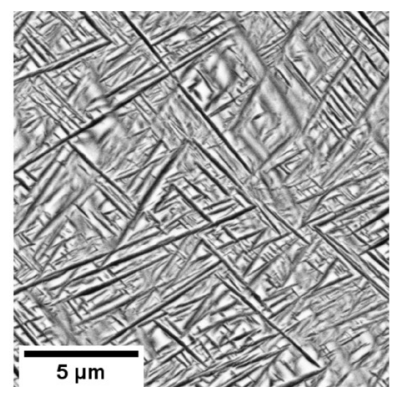

b)

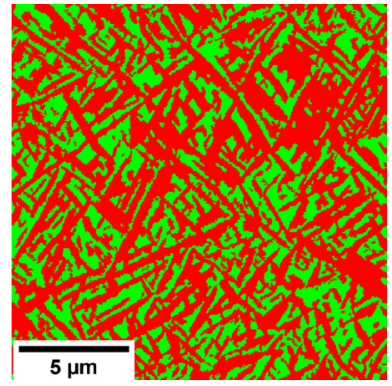

Fig. 3: Ti-5553 rapidly cooled from $600{ }^{\circ} \mathrm{C}$ to room temperature after 3 hours holding a) SEM/BSE image; b) corresponding EBSD phase map (red: $\alpha$-phase, green: $\beta$-phase)

The calibration curve between change in velocity and $\alpha$-phase fraction is shown in Fig. 4. They are expressed in terms of average quantities, in which the error bars are the velocity range at a particular time among repeated tests, and the $95 \%$ confidence interval of the metallographic data, respectively. The velocity change increases in a first approximation linearly with the $\alpha$-phase fraction, i.e. as proposed by Kruger and Damm [16] for the austenite-ferrite transformation in steels. With the labor-intensive metallographic measurements the phase fractions can be measured with a precision of $\pm 1 \%$ whereas with LUMet the fraction can be monitored with a precision of $\pm 6 \%$. Considering the advantages of the in-situ and non-contact nature of LUMet, this may be considered an excellent monitoring capacity for tracking phase transformations in Ti-alloys.

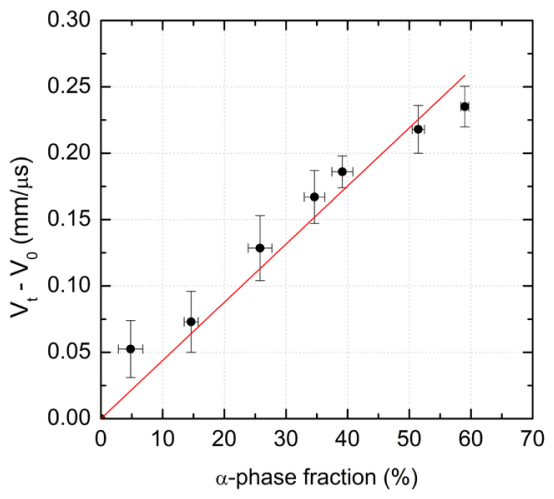

Fig. 4: Change in ultrasound velocity in Ti-5553 during isothermal aging at $600{ }^{\circ} \mathrm{C}$ as a function of $\alpha$-phase fraction

\section{Conclusion}

In-situ laser ultrasonics technique has been used to measure phase transformation kinetics in a Ti-5553 alloy. Using a systematic procedure to quantify $\alpha$-phase fractions from BSE images, a calibration curve has been created from which a linear relationship between change in longitudinal wave velocity and $\alpha$-phase fractions has been found to be a good approximation similar to previous findings for steels [16]. In the present study that was limited to one isothermal aging condition, i.e. at $600{ }^{\circ} \mathrm{C}$, the transformed fraction could be measured with an accuracy of $\pm 6 \%$ using LUMet. Further investigations are currently being conducted to extend these measurements to different aging temperatures as well as continuous cooling and heating treatments. Overall, laser ultrasonics is a promising technique for a real-time phase monitoring in industrial Ti-alloys.

\section{$\underline{\text { Acknowledgements }}$}

The authors thank Dr. Chad Sinclair for valuable discussions throughout this work and gratefully acknowledge the Natural Sciences and Engineering Research Council of Canada (NSERC) for the financial support provided.

\section{$\underline{\text { References }}$}

[1] C. J. Williams and G. Lütjering, Titanium, Berlin Heidelberg: Springer, 2007.

[2] J. D. Cotton, R. D. Briggs, R. R. Boyer, S. Tamirisakandala, P. Russo, N. Shchetnikov and J. C. Fanning, JOM, 67 (2015) 
1281-1303.

[3] R. R. Boyer and R. D. Briggs, J Mater Eng Perform, 14 (2005) 681-685.

[4] S. Shekhar, R. Sarkar, S. K. Kar and A. Bhattacharjee, Mater Design, 66 (2015) 596-610.

[5] S. Sadeghpour, S. M. Abbasi, M. Morakabati and S. Bruschi, Mater Design, 121 (2017) 24-35.

[6] F. Chen, G. Xu and K. Z. X. Zhang, Metall and Mat Trans A, 47 (2016) 5383-5394.

[7] W. Szkliniarz and G. Smotka, J Mater Process Tech, 53 (1995) 413-422.

[8] R. Dąbrowski, Arch Metall Mater, 56 (2011) 703-707.

[9] F. Bruneseaux, E. Aeby-Gautier, G. Geandier, J. D. C. Teixeira, B. Appolaire, P. Weisbecker and A. Mauroe, Mater Sci Eng A, 476 (2008) 60-68.

[10] S. Malinov, W. Sha, Z. Guo, C. Tang and A. Long, Mater Charact, 48 (2002) 279-295.

[11] D. Ma, A. Stoica, K. An, L. Yang, H. Bei, R. Mills, H. Skorpenske and X.-L. Wang, Metall and Mat Trans A, 42 (2011) 1444-1448.

[12] S. Malinov, P. Markovsky, W. Sha and Z. Guo, J Alloys Compd, 314 (2001) 181-192.

[13] J. D. C. Teixeira, B. Appolaire, E. Aeby-Gautier, S. Denis, G. Cailletaud and N. Späth, Mater Sci Eng A, 448 (2007) 135145.

[14] M. Falkenström, M. Engman, E. Lindh-Ulmgren and B. Hutchinson, Nondestruct Test Eval, 26 (2011) 237-252.

[15] M. Dubois, A. Moreau and J. F. Bussière, J Appl Phys, 89 (2001) 6487.

[16] S. E. Kruger and E. B. Damm, Mater Sci Eng A, 425 (2006) 238-243.

[17] S. Zamiri, B. Reitinger, H. Grun, J. Roither, S. Bauer, P. Burgholzer, IEEE, 2013.

[18] A. Shinbine, T. Garcin and C. Sinclair, Mater Charact, 117 (2016) 57-64.

[19] I. Hinterlechner, P. Barriobero-Vila, B. Reitinger, T. Fromherz, G. Requena and P. Burgholzer, Nondestruct Test Eval, 33 (2018) 130-138.

[20] B. A. Auld, Acoustic Fields and Waves in Solids, New York: John Wiley \& Sons Inc, 1973.

[21] J. D. Cotton, R. R. Boyer, R. D. Briggs, R. G. Baggerly, C. A. Meyer, M. D. Carter, W. Wood, G. Tewksbury, V. Li, X. Yao, Titanium 2007: Science and Technology, 2007.

[22] A. Shinbine, Master's thesis, The University of British Columbia, 2016.

[23] E. Collings, The Physical Metallurgy of Titanium Alloys, OH: American Society for Metals, Metals Park, 1984.

[24] S. Nag, R. Banerjee, R. Srinivasan, J. Y. Hwang, M. Harper and H. L. Fraser, Acta Mater, 57 (2009) 2136-2147.

[25] S. R. Sternberg, Computer, 16 (1983) 22-34.

[26] S. Wright, M. Nowell and J. Bingert, Metall and Mat Trans A, 38 (2007) 1845-1855.

[27] A. Moreau, "Laser-Ultrasonics for Metallurgy: Software and Applications," National Research Council Canada, 2012.

[28] E. Lee, R. Banerjee, S. Kar, D. Bhattacharyya and H. L. Fraser, Philos Mag, 87 (2007) 3615-3627. 